\title{
Rapid Beam Forming in Smart Antennas Using Smart-Fractal Concepts Employing Combinational Approach Algorithms
}

\author{
Mounissamy Levy, ${ }^{1}$ Sumanta Bose, ${ }^{1}$ D. Sriram Kumar, ${ }^{1}$ and Anh Van Dinh ${ }^{2}$ \\ ${ }^{1}$ Department of Electronics and Communication Engineering, National Institute of Technology Trichy, Tamil Nadu 620015, India \\ ${ }^{2}$ Department of Electrical and Computer Engineering, University of Saskatchewan, Saskatoon, SK, Canada S7N 5A9
}

Correspondence should be addressed to Mounissamy Levy, 408110003@nitt.edu

Received 6 February 2012; Revised 9 September 2012; Accepted 9 September 2012

Academic Editor: Dalia N. Elshiekh

Copyright ( 2012 Mounissamy Levy et al. This is an open access article distributed under the Creative Commons Attribution License, which permits unrestricted use, distribution, and reproduction in any medium, provided the original work is properly cited.

\begin{abstract}
Smart antennas offer a broad range of ways to improve wireless system performance. They provide enhanced coverage through range extension, hole filling, and better building penetration. Smart antennas use an array of low gain antenna elements which are connected by a network. Fractal concepts have been used in antenna arrays recently. The important properties of fractal arrays are frequency independent multiband characteristics, schemes for realizing low side lobe designs, systematic approaches to thinning, and the ability to develop rapid beam forming algorithms. In this paper, an attempt has been made to apply assignment of usage time and location tag algorithm for smart antennas combined with the fractal concepts to reduce the computational complexity and enhance resource allocation for rapid beam forming algorithms. Furthermore, two combinational approach algorithms are proposed for peer users within single base station and peer users between different base stations.
\end{abstract}

\section{Introduction}

Smart antennas are MIMO arrays that emphasize the signal of interest and minimizes the interfering signals by adjusting or adapting its own beam pattern. This is done by varying the relative phases of the respective signals feeding the antennas in such a way that the effective radiation pattern of the array is reinforced in the desired direction and suppressed in undesired directions to model any desired radiation pattern. Smart antenna techniques are used notably in signal processing, RADAR, radio astronomy, and cellular systems like $\mathrm{W}$ CDMA and UMTS. The smart antenna concept can be used in optical antenna technology also to produce rapid beam scanning. Spatial time multiplexing techniques and space time block code techniques also widely use smart antennas. UWB communication also makes use of smart antennas with proper bandwidth allocated to it.

\section{Fractal Concepts}

A fractal is a recursively generated object having a fractional dimension. Many objects, including antennas, can be designed using the recursive nature of a fractal. The important properties of fractal arrays are frequency independent multiband characteristic schemes for realizing low-side lobe designs, systematic approaches to thinning, and the ability to develop rapid beam-forming algorithms by exploiting the recursive nature of fractals. These arrays have fractional dimensions that are found from generating subarray used to recursively create the fractal array.

The term fractal, meaning broken or irregular fragments, was originally coined by Mandelbrot [1] to describe a family of complex shapes that possess an inherent self-similarity in their geometrical structure. Since the pioneering work of Mandelbrot and others, a wide variety of applications for fractals have been found in many branches of science and engineering. One such area is fractal electrodynamics, in which fractal geometry is combined with electromagnetic theory for the purpose of investigation of new class of radiation, propagation, and scattering problems. One of the most promising areas of fractal electrodynamics research is in its applications to antenna theory and design. We refer to this new and rapidly growing field of research as fractal antenna engineering. There are primarily two active areas 
of research in fractal antenna engineering: study of fractalshaped antenna elements and the use of fractals in antenna arrays [2-4].

The first application of fractals to the field of antenna theory was reported by Kim and Jaggard [5]. They introduced a methodology for designing low-side lobe arrays which is based on the theory of random fractals. Lakhtakia et al. [6] demonstrated that the diffracted field of a self-similar fractal screen also exhibits self-similarity. The fact that selfscaling arrays can produce fractal radiation patterns was first established and the work was later extended to the case of concentric ring arrays by Liang et al. [7]. Applications of fractal concepts to the design of multiband Koch arrays, as well as to low-side lobe Cantor arrays, are discussed by Puente-Baliarda and Pous [8]. Other types of fractal array configurations are discussed by Werner et al. [9].

A rich class of fractal arrays exists which can be formed recursively through the repetitive application of a generating subarray. A generating subarray is a small array at scale one $(P=1)$ where $P$ is the scale factor and is used to construct larger arrays at higher scales (i.e., $P>1$ ). In many cases, the generating subarray has elements that are turned on and off in a certain pattern. A set formula for copying, scaling, and translation of the generating subarray is then followed in order to produce the fractal array. Hence, fractal arrays that are created in this manner will be composed of a sequence of self-similar subarrays. In other words, they may be conveniently thought of as arrays of arrays [9].

The array factor for a fractal array of this type may be expressed in the general form:

$$
\operatorname{AF}_{P}(\psi)=\prod_{i=1}^{P} \widehat{\mathrm{GA}}\left(\delta^{i-1} \psi\right),
$$

where $\mathrm{GA}(\psi)$ represents the array factor associated with the generating subarray. The parameter $\delta$ is a scale or expansion factor that governs how large the array grows with each recursive application of the generating subarray. The expression for the fractal array factor given in (1) is simply the product of scaled versions of a generating subarray factor. Therefore, we may regard equation (1) as representing a formal statement of the pattern multiplication theorem for fractal arrays. Applications of this specialized pattern-multiplication theorem to the analysis and development of rapid beam forming algorithms will be considered in the following sections.

\section{Smart Antennas: Beam Forming}

There is an ever-increasing demand on mobile wireless operators to provide voice and high-speed data services. At the same time, these operators want to support more users per base station to reduce overall network costs and make the services affordable to subscribers. As a result, wireless systems that enable higher data rates and higher capacities are a pressing need. Unfortunately, because the availability of broadcast spectrum is limited, attempts to increase traffic within a fixed bandwidth create more interference in the system and degrade the signal quality [10-12].
In particular, when Omni-directional antennas, as shown in Figure 1(a) [13] are used at the base station, the transmission/reception of each user's signal becomes a source of interference to other users located in the same cell, making the overall system interference limited. An effective way to reduce this type of interference is to split up the cell into multiple sectors and use sectorized antennas, as shown in Figure 1(b) [13].

Smart antenna technology offers a significantly improved solution to reduce interference levels and improve the system capacity. With this technology, each user's signal is transmitted and received by the base station only in the direction of that particular user. This drastically reduces the overall interference in the system. A smart antenna system, as shown in Figures 2 and 3 [13], consists of an array of antennas that together direct different transmission/reception beams towards each user in the system. This method of transmission and reception is called beam forming and is made possible through smart (advanced) signal processing at the baseband.

In beam forming, each user's signal is multiplied with complex weights that adjust the magnitude and phase of the signal to and from each antenna. This causes the output from the array of antennas to form a transmit/receive beam in the desired direction and minimizes the intensities in other directions.

If the complex weights are selected from a library of weights that form beams in specific, predetermined directions, the process is called switched beam forming. Here, the base station basically switches between the different beams based on the received signal strength measurements. On the other hand, if the weights are computed and adaptively updated in real time, the process is called adaptive beam forming. Through adaptive beam forming, the base station can form narrower beams towards the desired user and nulls towards interfering users, considerably improving the signalto-interference-plus-noise ratio.

\section{Fractal Algorithm}

One of the more intriguing attributes of fractal arrays is the possibility for developing algorithms, based on the compact product representation of (1), which are capable of performing extremely rapid pattern computations. For example consider a linear array of isotropic elements, uniformly spaced and a distance $d$ apart along the $z$ axis. The array factor corresponding to this linear array may be expressed in the form:

$$
\operatorname{AF}(\psi)=I_{\mathrm{o}}+2 \sum_{n=1}^{N} I_{n} \cos \{n \psi\}
$$

for odd number of elements and

$$
\operatorname{AF}(\psi)=2 \sum_{n=1}^{N} I_{n} \cos \left\{\left(n-\frac{1}{2}\right) \psi\right\}
$$




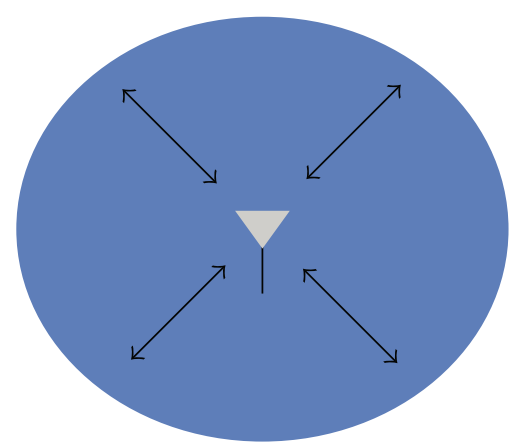

(a) Omnidirectional

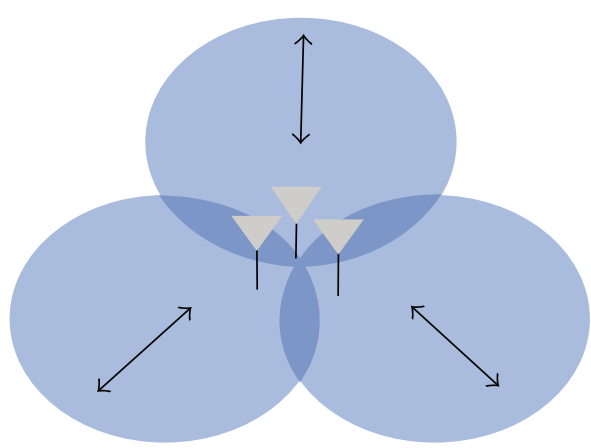

(b) Sectorized

Figure 1: Non-smart (conventional) antenna system.

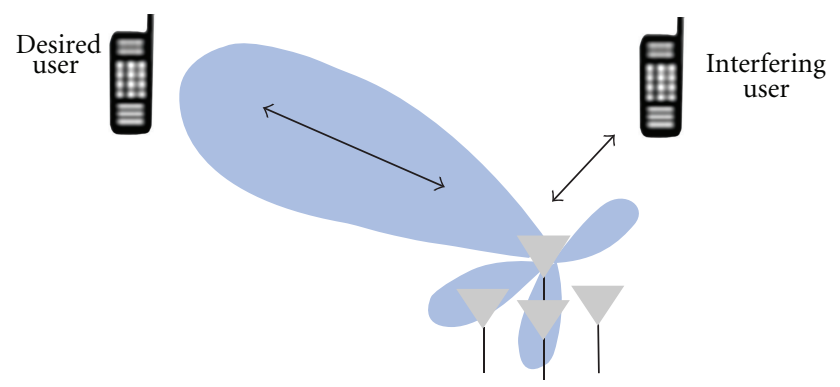

FIGURE 2: Smart antenna system-beam forming.

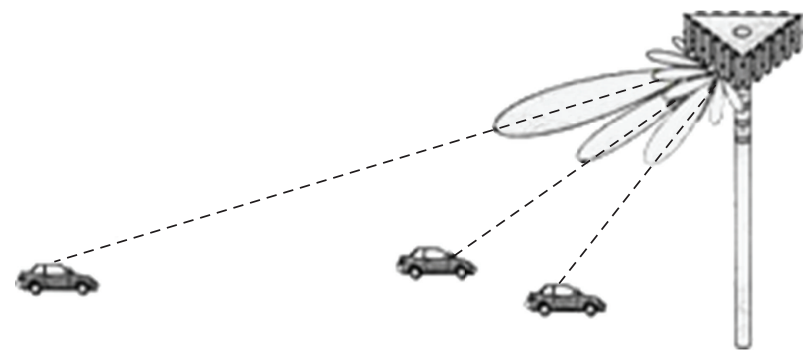

FIGURE 3: Separate beam for each user.

for even number of elements, where $n$ is the total number of elements:

$$
\begin{gathered}
N=\left(\frac{n-1}{2}\right), \\
\psi=k d\left\{\cos \theta-\cos \theta_{0}\right\}, \\
k=\frac{2 \pi}{\lambda} .
\end{gathered}
$$

The directivity for fractal array antenna is given by

$$
D_{P}(u)=\frac{\widehat{\mathrm{AF}_{P}^{2}}((\pi / 2) u)}{(1 / 2) \int_{-1}^{1} \widehat{\mathrm{AF}_{P}^{2}}((\pi / 2) u) d u},
$$

where

$$
\begin{gathered}
\psi=\frac{\pi}{2} u, \\
u=\cos \theta .
\end{gathered}
$$

These arrays become fractal-like when appropriate elements are turned off or removed, such that

$$
\begin{aligned}
& I_{n}=1, \text { if element } \mathrm{n} \text { is turned ON, \& } \\
& I_{n}=0, \text { if element } \mathrm{n} \text { is turned OFF. }
\end{aligned}
$$

Hence, fractal arrays produced by following this procedure belong to a special category of thinned arrays. If the above equations are used to calculate the array factor for an odd number of elements, then $N$ cosine functions must be evaluated and $N$ additions performed, for each angle. One of the simplest schemes for constructing a fractal linear array follows the recipe for the Cantor set. Cantor linear arrays were first proposed and studied in [8] for their great potential use in the design of low-side lobe arrays.

The basic triadic Cantor array may be created by starting with a three-element generating subarray, and then applying it repeatedly over $P$ scales of growth. The generating subarray in this case has three uniformly spaced elements, with the center element turned off or removed, that is, 101 . The triadic Cantor array is generated recursively by replacing 1 by 101 and 0 by 000 at each stage of the construction. For example, at the second stage of construction $(P=2)$, the array pattern would look like

$$
1010000101 .
$$

In this fashion the different stages of fractal pattern are grown. Starting with the basic stage whatever value we assume for the basic stage the value will be substituted for each stage and the antenna array grows in size rapidly. The same case can be applied for planar construction also. Current research and investigations on three-dimensional fractal arrays are in progress to refine and tailor the pattern of the required beam. Research is also in progress on nonlinear arrays with nonuniform amplitude and unequal spacing arrays to get the radiation pattern of desired extent.

At the third stage $(P=3)$, we would have

$$
101000101000000000101000101 \text {. }
$$

The array factor of the three-element generating subarray with the representation 101 is:

$$
\mathrm{GA}(\psi)=2 \cos \{\psi\}
$$




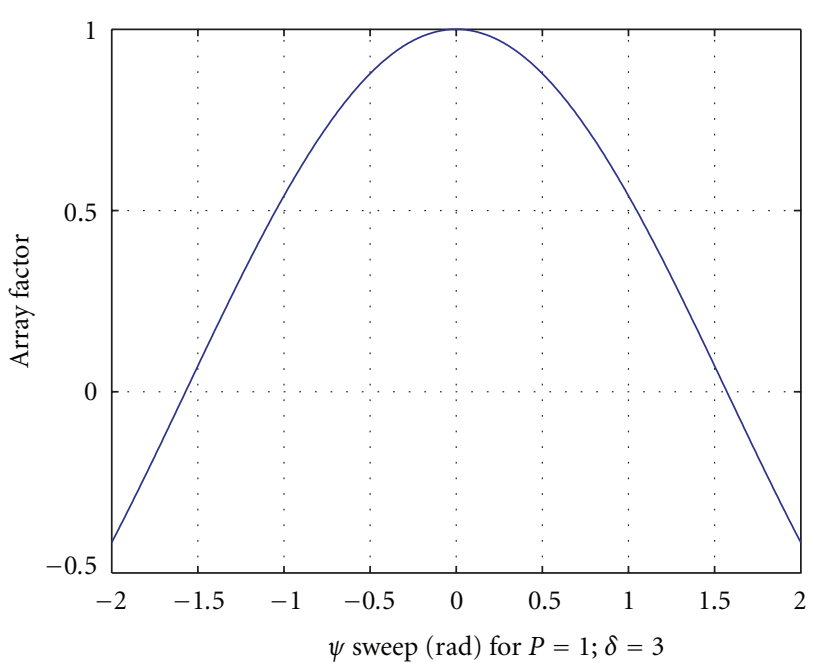

FIgURE 4: Fractal array factor pattern for $P=1 ; \delta=3$.

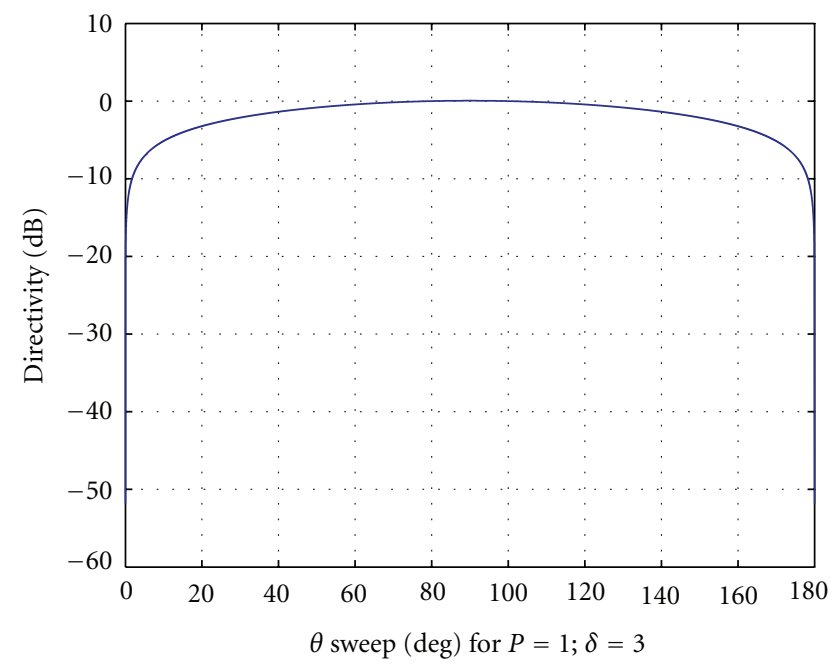

Figure 5: Directivity pattern for $P=1 ; \delta=3$.

which may be derived from the array factor equation by setting $N=1, I_{0}=0$, and $I_{1}=1$. Substituting this equation into equation (1) and choosing an expansion factor of 3 , result in an expression for the Cantor array factor given by

$$
\widehat{\mathrm{A}} \mathrm{F}_{P}(\psi)=\prod_{i=1}^{P} \hat{\mathrm{G}} \mathrm{A}\left(3^{i-1} \psi\right)=\prod_{i=1}^{P} \cos \left\{3^{i-1} \psi\right\} .
$$

Here the hat notation indicates that the quantities have been normalized. The array factor pattern and the directivity pattern for $P \in\{1,2,3,4\}$ and $\delta=3$ are shown in Figures 4, $5,6,7,8,9,10$, and 11 .

As both values increase the plot shows improvement in characteristics, the directivity increases, and the pattern becomes narrower. Another fantastic advantage is that equation (10) only requires $P$ cosine-function evaluations and $P-1$ multiplication. In the case of an 81 element triadic cantor array, the fractal array factor is at

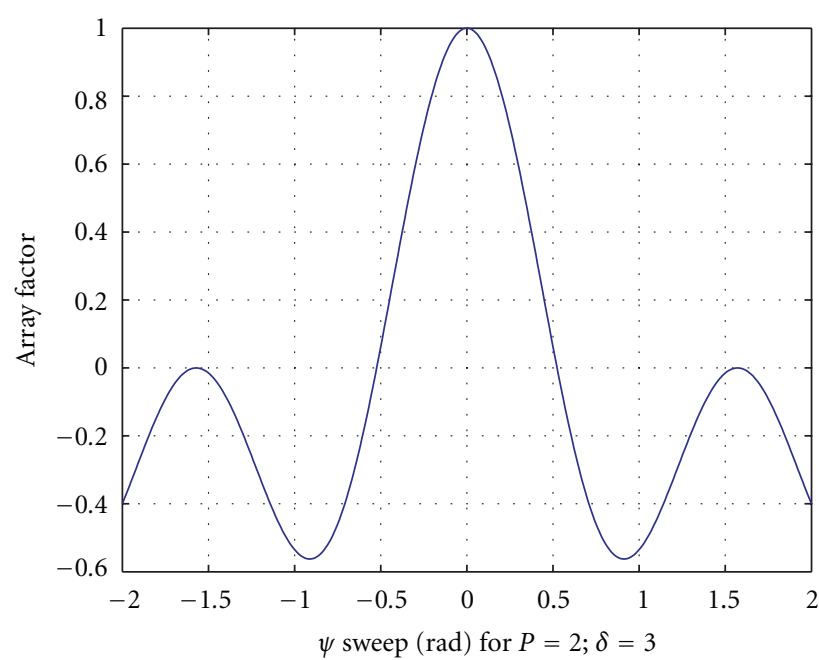

Figure 6: Fractal array factor pattern for $P=2 ; \delta=3$.

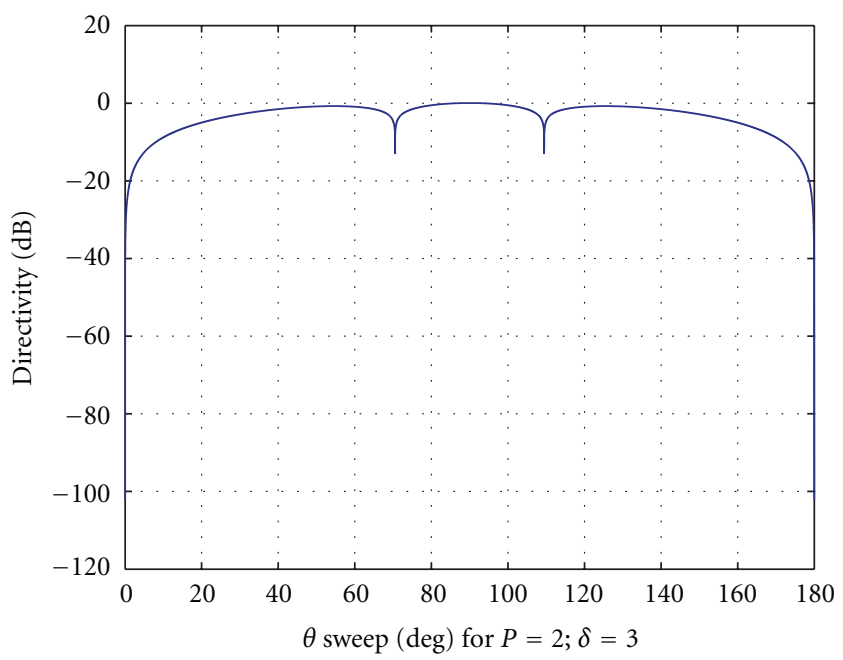

Figure 7: Directivity pattern for $P=2 ; \delta=3$.

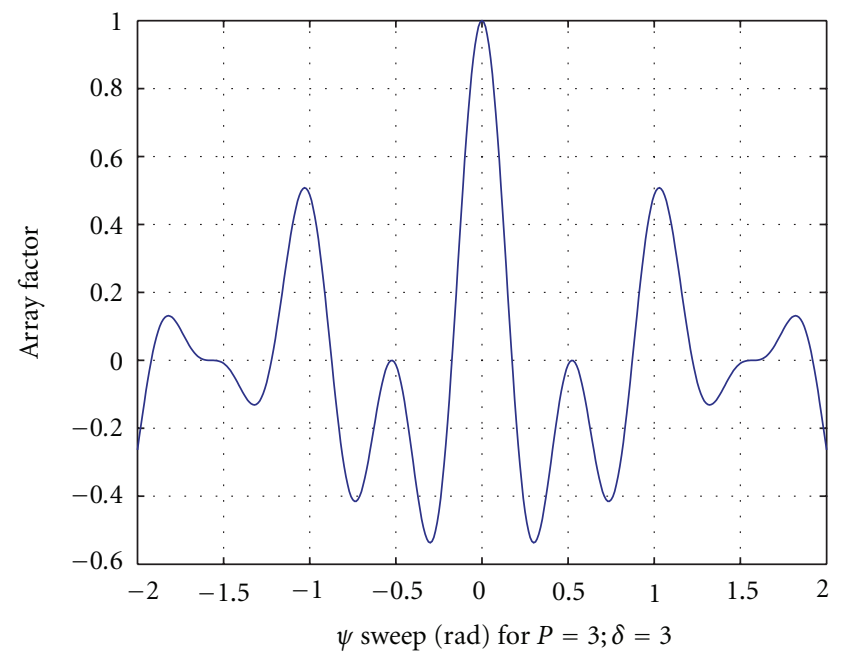

Figure 8: Fractal array factor pattern for $P=3 ; \delta=3$. 


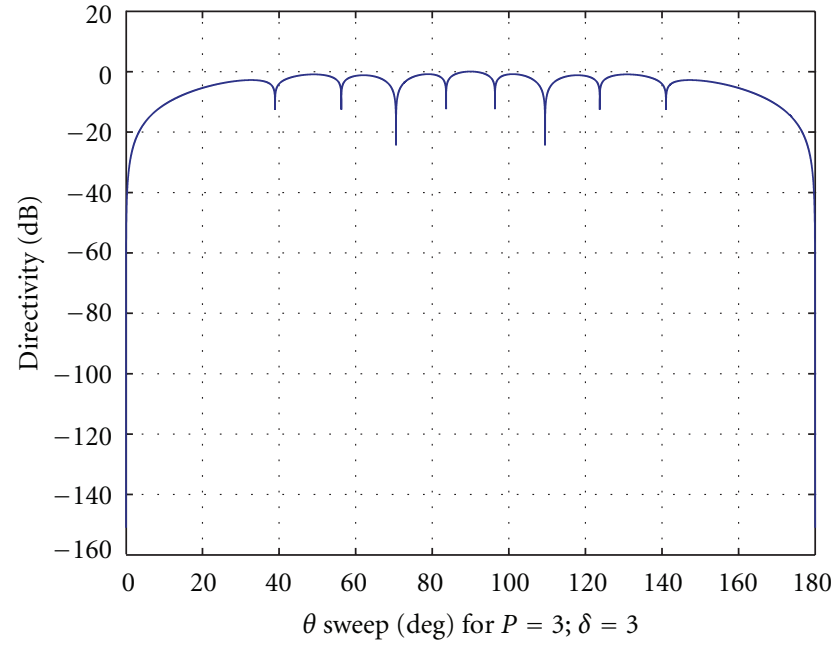

Figure 9: Directivity pattern for $P=3 ; \delta=3$.

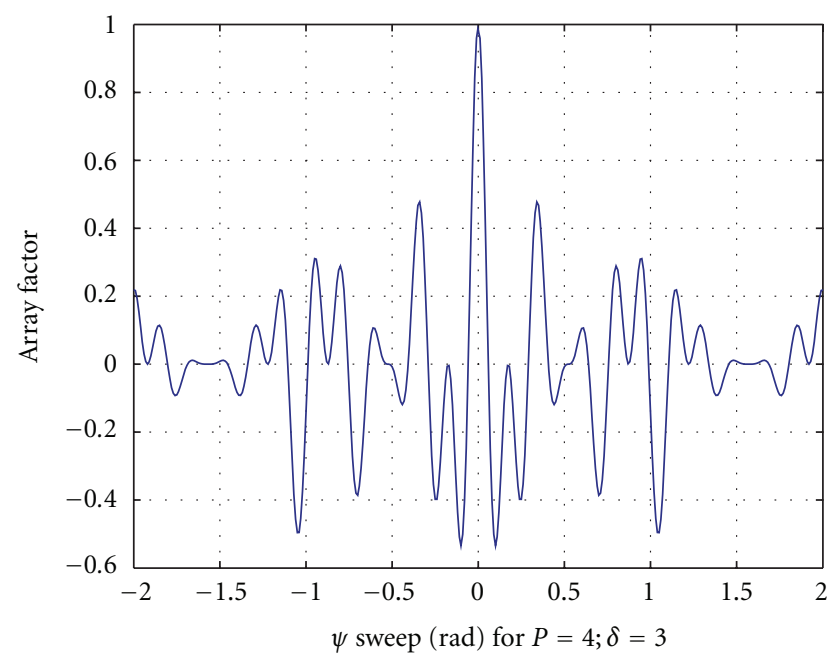

FIgURE 10: Fractal array pattern for $P=4 ; \delta=3$.

least $N / P=40 / 4=10$ times faster to calculate than the conventional discrete Fourier transform. The multiband characteristics of linear fractal array are discussed in $[6,7]$. The same procedure can be applied for the Sierpinski carpet arrays for developing efficient algorithms which can be used in planar smart antennas. The multiband characteristics of Sierpinski carpet array are discussed in [8]. More about fractal arrays are discussed in [9].

Table 1 shows the increase in speed factor as the values of $P$ and $\delta$ vary. As the value of $P$ and $\delta$ increases the speed factor increases enormously. For example, with for $P=4$ and $\delta=7$ the fractal array factor is 300 times faster to calculate than the conventional case. This property can be utilized in smart antennas to produce rabid beam forming algorithms.

\section{Assignment of Priorities Algorithm}

After studying the advantages of fractal concepts over the development of rapid beam forming algorithms in smart

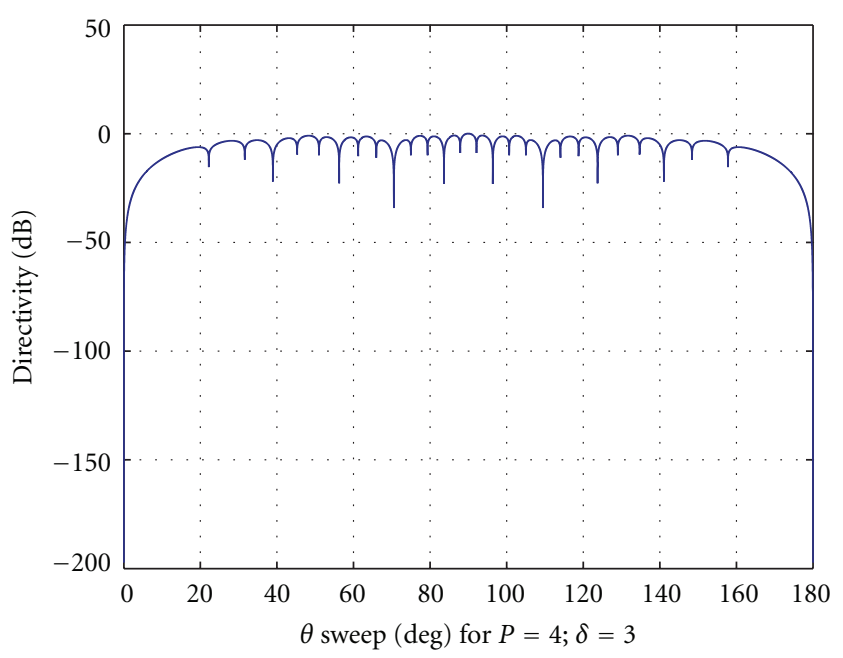

Figure 11: Directivity pattern for $P=4 ; \delta=3$.

TABle 1: Tabulation of the variation of $P$ and $\delta$.

\begin{tabular}{lccccc}
\hline & $P=1$ & $P=2$ & $P=3$ & $P=4$ & $P=5$ \\
\hline$\delta=3$ & 1 & 2 & 4 & 10 & 24 \\
$\delta=5$ & 2 & 6 & 20 & 78 & 312 \\
$\delta=7$ & 3 & 12 & 57 & 300 & 1680 \\
$\delta=9$ & 4 & 20 & 121 & 820 & 5904 \\
\hline
\end{tabular}

TABLE 2: Initial look-up table.

\begin{tabular}{lcccc}
\hline Sl no. & $\begin{array}{c}\text { User } \\
\text { beam }\end{array}$ & $\begin{array}{c}\text { Priority } \\
\text { assigned }\end{array}$ & $\begin{array}{c}\text { Computational } \\
\text { complexity }\end{array}$ & $\begin{array}{c}\text { Needed } \\
\text { resource } \\
\text { allocation }\end{array}$ \\
\hline 1 & A & 1 & High & High \\
2 & B & 2 & Medium & Medium \\
3 & C & 3 & Low & Low \\
4 & D & 4 & Very low & Very low \\
\hline
\end{tabular}

antennas, now we can propose a new algorithm technique of assigning priorities for each beam. Let us consider four users. Initially, each user is assigned an individual beam from smart antenna with uniform distribution. Consider the situation that user A is moving very fast, user B is medium speed, user C is low speed, and user D is pedestrian, as illustrated in Table 2. With these different speed users we can assign a new priority for each user with the beam.

Since user A is rapidly moving his beam needs more computation complexity than user B. Similarly user C needs less computation complexity than user B. Likewise, since user D is a pedestrian he needs even less computational complexity than used C. Now let us introduce a token passing technique between the users beam. The token named " $\mathrm{X}$ " mainly indicates the computational complexity effect. Initially let us consider the token is captured by the user D. The token X looks at the priority of user D. His priority is 4 . The token checking algorithm will check for the next least number having the highest priority. Subsequently it will find that user $\mathrm{C}$ has a priority 3 . Similarly after the second 
TABLE 3: Token X caught by user D on random.

\begin{tabular}{lccccc}
\hline Sl no. & $\begin{array}{c}\text { User } \\
\text { beam }\end{array}$ & $\begin{array}{c}\text { Priority } \\
\text { assigned }\end{array}$ & $\begin{array}{c}\text { Computational } \\
\text { complexity }\end{array}$ & $\begin{array}{c}\text { Resource } \\
\text { allocation }\end{array}$ & Token \\
\hline 1 & A & 1 & Uniform & Uniform & \\
2 & B & 2 & Uniform & Uniform & \\
3 & C & 3 & Uniform & Uniform & \\
4 & D & 4 & Uniform & Uniform & X \\
\hline
\end{tabular}

TABLE 4: Token X caught by user $\mathrm{C}$ after first iteration.

\begin{tabular}{lccccc}
\hline Sl no. & $\begin{array}{c}\text { User } \\
\text { beam }\end{array}$ & $\begin{array}{c}\text { Priority } \\
\text { assigned }\end{array}$ & $\begin{array}{c}\text { Computational } \\
\text { complexity }\end{array}$ & $\begin{array}{c}\text { Resource } \\
\text { allocation }\end{array}$ & Token \\
\hline 1 & A & 1 & Uniform & Uniform & \\
2 & B & 2 & Uniform & Uniform & \\
3 & C & 3 & Uniform & Uniform & X \\
4 & D & 4 & Low & Low & \\
\hline
\end{tabular}

TABLE 5: Token X caught by user B after second iteration.

\begin{tabular}{lccccc}
\hline Sl no. & $\begin{array}{c}\text { User } \\
\text { beam }\end{array}$ & $\begin{array}{c}\text { Priority } \\
\text { assigned }\end{array}$ & $\begin{array}{c}\text { Computational } \\
\text { complexity }\end{array}$ & $\begin{array}{c}\text { Resource } \\
\text { allocation }\end{array}$ & Token \\
\hline 1 & A & 1 & Uniform & Uniform & \\
2 & B & 2 & Uniform & Uniform & X \\
3 & C & 3 & Low & Low & \\
4 & D & 4 & Very low & Very low & \\
\hline
\end{tabular}

iteration it will see the user B who is having still higher priority. Then the token will be passed to B. After the next iteration the user $\mathrm{A}$ is found to be having the highest priority among others and finally the token will be passed to A. The result after each iteration process is shown in the Tables 3, 4, 5, and 6 clearly. Since A is moving very fast, he needs highest computational complexity so that more computational resources can be allotted to the particular user compared to others and the rapid beam forming technique can be still enhanced. The tables illustrate this process in a step-by-step manner. Coverage performance analysis is discussed in [14] by Badjian et al. and uses genetic algorithm technique. But the proposed one in this paper is a novelistic approach to reduce the computational complexity and properly allocate the resource so that rapid beam forming can be enhanced.

\section{Assignment of Scanning Beams Algorithm}

Smart antenna technology uses the concept of locating the user beam and adaptive algorithms are used to adaptively trace the user along with the beam. For accomplishing this task efficiently so many adaptive algorithms are available. An alternative approach to this technology is scan antenna technology in which beams are allowed to scan randomly and depending upon the speed of the user, each user is allocated a scanning beam. This concept is new and greatly reduces the time needed for computation and thereby increasing the rapidness of rapid beam and saving the time needed for
TABLE 6: Token X caught by user A after third iteration matches exactly with the look-up table.

\begin{tabular}{lccccc}
\hline Sl no. & $\begin{array}{c}\text { User } \\
\text { beam }\end{array}$ & $\begin{array}{c}\text { Priority } \\
\text { assigned }\end{array}$ & $\begin{array}{c}\text { Computational } \\
\text { complexity }\end{array}$ & $\begin{array}{c}\text { Resource } \\
\text { allocation }\end{array}$ & Token \\
\hline 1 & A & 1 & High & High & X \\
2 & B & 2 & Medium & Medium & \\
3 & C & 3 & Low & Low & \\
4 & D & 4 & Very low & Very low & \\
\hline
\end{tabular}

TABLE 7: Initial lookup table with different user speed.

\begin{tabular}{lcccc}
\hline Sl no. & User & $\begin{array}{c}\text { Moving } \\
\text { speed }\end{array}$ & $\begin{array}{c}\text { Computational } \\
\text { complexity }\end{array}$ & $\begin{array}{c}\text { Resource } \\
\text { allocation }\end{array}$ \\
\hline 1 & A & Very fast & High & High \\
2 & B & Medium & Medium & Medium \\
3 & C & Low & Low & Low \\
4 & D & Pedestrian & Very low & Very low \\
\hline
\end{tabular}

TABLE 8: Initial scanning beam table from scan antenna.

\begin{tabular}{lcccc}
\hline Sl no. & $\begin{array}{c}\text { Scanning } \\
\text { beam }\end{array}$ & $\begin{array}{c}\text { Moving } \\
\text { speed }\end{array}$ & $\begin{array}{c}\text { Computational } \\
\text { complexity }\end{array}$ & $\begin{array}{c}\text { Needed resource } \\
\text { allocation }\end{array}$ \\
\hline 1 & 1 & Very fast & High & High \\
2 & 2 & Medium & Medium & Medium \\
3 & 3 & Low & Low & Low \\
4 & 4 & Pedestrian & Very low & Very low \\
\hline
\end{tabular}

TABLE 9: Matching algorithm matches the speed of beam and speed of user.

\begin{tabular}{lccc}
\hline Sl no. & Scanning beam & User & Moving speed \\
\hline 1 & 1 & A & Very fast \\
2 & 2 & B & Medium \\
3 & 3 & C & Low \\
4 & 4 & D & Pedestrian \\
\hline
\end{tabular}

resource allocation. Let us consider the same case of four users. User A is moving very fast, user B is of medium speed, user C is of low speed, and user D is pedestrian. Initially the scan antenna technology produces four different beams independently at random speed. Let the four beams be named beam 1, beam 2, beam 3, and beam 4 , respectively. Consider the situations such that beam 1 is moving very fast, beam 2 is of medium speed, beam 3 is of low speed, and beam 4 is of very low speed. Now a matching technique has to be used to carefully match the speed of the user with the speed of the beam. Once the matching has been done, then each user can lock with his beam as long as he uses it and release the beam free after the use so that other users can use the beam. Tables 7,8 , and 9 illustrate this process in a step-by-step manner.

\section{Assignment of Usage Time and Location Tag Algorithm}

While the advantages of fractal concepts over the development of rapid beamforming algorithms in smart antennas 
TABLE 10: Look-up table for assignment of usage time and location tag.

\begin{tabular}{lcccc}
\hline Sl no. & User & $\begin{array}{c}\text { Usage time } \\
(\tau)\end{array}$ & $\begin{array}{c}\text { Location tag } \\
(\sigma)\end{array}$ & $\begin{array}{c}\text { Computation and } \\
\text { resource allocation }\end{array}$ \\
\hline 1 & A & Long duration & Slow moving & Very low \\
2 & B & Long duration & Fast moving & Low \\
3 & C & Short duration & Slow moving & Medium \\
4 & D & Short duration & Fast moving & High \\
\hline
\end{tabular}

using new algorithms have been studied by the authors in $[15,16]$, the concept can now be extended to another new algorithm technique called "Assignment of Usage Time and Location Tag Algorithm." Let us consider the same case of four users. Assume that the initial two proposed algorithms are applied and the system is working in efficient mode. We will simply call this as efficient mode as far as our case is considered. This can be considered as algorithm over algorithms to increase the effectiveness of the system. Now let us introduce the parameters called $\tau$ which refers to the effective usage time of each user and $\sigma$ which refers to the location tag of each user. Assume that we have some mechanisms to know the probability of usage duration of each user and we got the location tag by global positioning systems. Note that we have already optimized the system with respect to user speed and having introduced a new technique called scan antenna technology; now these additional parameters can still enhance the system speed effectively. From the smart antenna side there is a considerable amount of time that is needed for the development of each new beam. With these two parameters, $\tau$ and $\sigma$, now there are four possible cases for each user if we take only two variables into account, that is, long and short duration user and fast and slow moving user. A user can be in long duration and slow moving, long duration and fast moving, short duration and slow moving, and finally short duration and fast moving as shown in Table 10.

From Table 10 it can be easily concluded that user A can be considered as stationary user since he is in long duration and slow moving. The term smart can be removed from user A antennas and can be allotted a single wide coverage beam antenna. The computational complexity time and resource allocation will be nullified. User B can be given less priority for he is taking long duration though moving fast. The type of beam pattern can be recognized and stored in memory. User $\mathrm{C}$ is taking short duration and moving slow, a type of medium complexity can that be allotted. And finally as User $\mathrm{D}$ is concerned, he is taking short duration and moving fast; therefore, more complexity is required for him. All this data can be observed for a long-time basis and stored in memory so that the observation database can help in allocating the resources which speeds up the process of producing rapid beams. Coverage performance analysis is discussed in [14] by Badjian et al. and uses genetic algorithm technique. But the proposed one in this paper is a novelistic approach to reduce the computational complexity and properly allocate the resource so that rapid beam forming can be enhanced.

\section{Combinational Beam-Forming Algorithm among Peer Members within a Single Base Station}

This is a new algorithm for the rapid beam forming in smart antennas which aims to bring different beams acquired at different times into geometric coincidence, possibly by different beam forming stations at different places, as shown in Figure 12. This aims at achieving time and space coincidence of various beams. Consider for example 12 users are using mobile phone at a time belonging to single cell served by a smart antenna. If each user is allocated a separate beam, then 12 different beams are required. The complexity of the system is increased and the time required for each beam will increase. The rapid beam forming will be still worsened if the number of user still increases. In this proposed new algorithm, the pattern of each user is observed for a specific amount of time and the users will be grouped in clusters say for example four clusters of three in each group, then the complexity can be reduced by a factor of four. In the first case, the cluster can be preknown numbers and in the extreme case the cluster can be assigned in random depending upon the number of users having the similar pattern. This pattern can be from a single base station or it can be from all the base stations forming a single hop among peer members.

The dramatic growth of the wireless communication industry has resulted in searches for new technologies to provide broader bandwidth per user channel, better quality, and new value added services. Employing smart antennas presents an elegant and relatively economical way to improve the performance of wireless transmission. Conditioned on the array factor of the form given in (1)-(3), fractal array is a sum of bounded independent and identically distributed complex random variables. Therefore by the strong law of large numbers for fixed, the resulting beam pattern should approach the ensemble average with the probability one as the number of antenna element increases.

However, in practice we are interested in finite relatively small values of $N$ and in this case the average beam pattern does not necessarily represent the beam pattern of any particular realization. We are interested in the average beam pattern of these two particular realizations of randomly generated antenna arrays. The main lobes of the realization beam patterns closely match the average, but the side lobes may fluctuate with a large dynamic range and their peaks often well exceed the average level. Therefore, in practice, the statistical distributions of beam patterns and sidelobes, in particular, are of importance. The block diagram illustrates the algorithm. In the first stage all the clustered beam groups are tabulated and a mapping is done between the similar beams. In the next stage the sampling of all the beams in space and time domain is done. The next stage detects the similar beams and passes it to the space and time detection block. Again it is passed to the correlation detector.

The residual fitting for the similar beams is done using any residual preferences. The cluster is grouped among peer members and from this block the feedback is given for the next iteration. As per the output from this block the resource is allocated efficiently and the rapid beam forming 


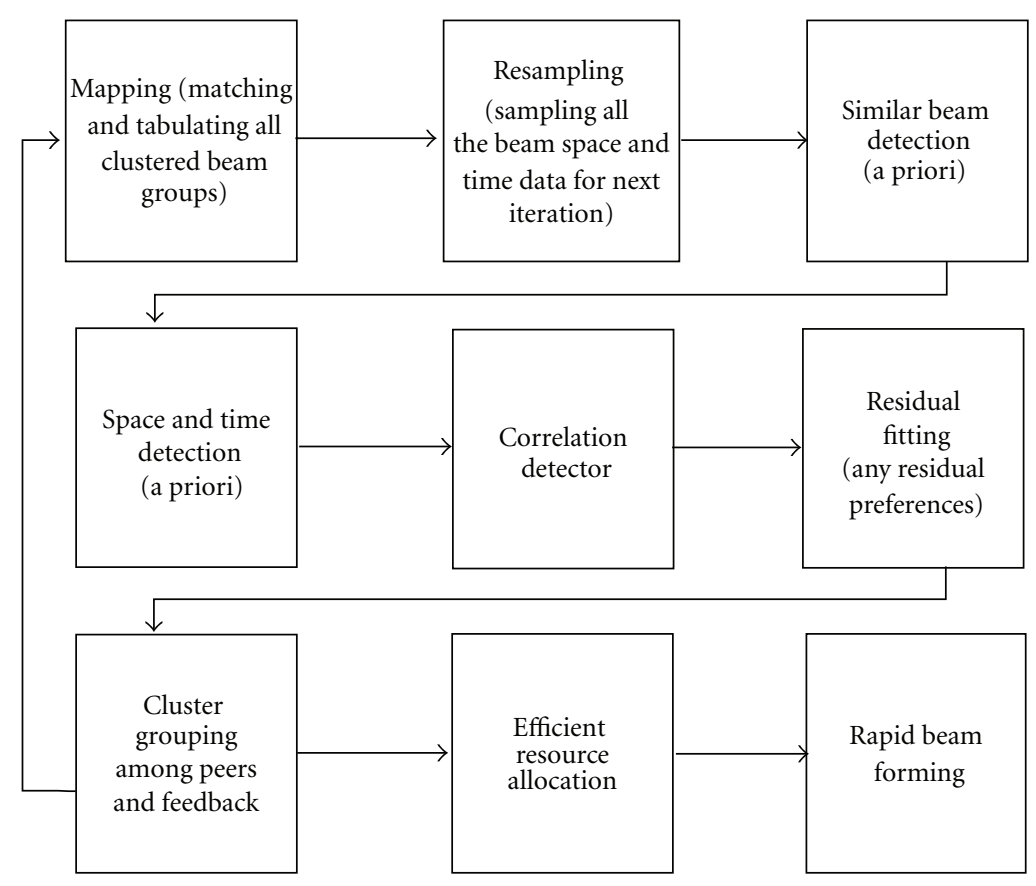

FIGURE 12: Block diagram for combinational beam-forming algorithm among peer members within a single base station.

is done. Different technical methods used can include Eigen combining algorithm, realistic beam forming algorithm, and maximal ratio combining. This signal processing methods can be employed for either receiving side or transmitting side smart antenna technology. The statistical beam forming techniques and maximal-ratio combining (MRC) techniques can be used along with conventional techniques. Due to array and diversity gain great performance benefits can be achieved in favorable propagating conditions. For unfavorable propagating conditions the performance can be little bit affected.

Furthermore although their numerical complexity remains fixed, the performance fluctuates when propagating conditions vary as in actual scenarios. Adaptive eigencombining can also considered for the beamforming. Maximal ratio eigencombining unifies the signal processing principles and analyses. The array signal processing has been applied for some decades as an attractive method for signal detection and estimation in hash environment. An array of sensors can be flexibly configured to exploit spatial and temporal characteristics of signal and noise and has many advantages over single sensor. This has many applications in radar, radio astronomy, sonar, wireless communication, seismology, speech acquisition, medical diagnosis and treatment, and so forth there are two kinds of array beamformers: fixed beamformer and adaptive beamformer. The weight of fixed beamformer is predesigned and it does not change in applications. The adaptive beamformer automatically adjusts its weight according to some criteria. It significantly outperforms the fixed beamformer in noise and interference suppression.

A typical representative is the linearly constrained minimum variance beamformer. A famous representative for linearly constrained minimum variance beamformer is the capon beamformer. In ideal cases the capon beamformer has high performance in interference and noise suppression provided that the array steering vector is known. The performance of the adaptive beamformers highly degrades when there are array imperfections such as steering direction error, time delay error, and phase errors of the array sensors, multipath propagation effects, and wave front distortions. This is known as the target signal cancellation problem. Tremendous work has been done to improve the robustness of adaptive beamformer. To overcome the problem of target signal cancellation caused by the steering direction error, multiple point constraints were introduced in adaptive array. The idea of this approach is intuitive. With multiple gain constraints at different directions in the vicinity of the assumed one, the array processor becomes robust in the region where constraints are imposed.

However the available number of constraints is limited because the constraints consume the degrees of freedoms (DOFs) of array processor for interference suppression. If compact antennas are used for fast beam forming then the parasitic array antennas are suitable for power consumption limited mobile terminal applications. Two categories of parasitic antennas are there, namely, switched parasitic array antennas and reactively controlled directive array. In comparison with switched parasitic array antennas, reactively controlled directive array can realize a smoother beam pattern steering, thus achieving a better interference suppression performance. The reactively controlled directive array antenna is an analog adaptive antenna. Adaptive beam forming is achieved by adaptively adjusting the load reactance of parasitic elements. When the DOA of the impinging interference signal is close to that of the desired signal, the null forming ability degrades. This is due to the high spatial correlation between the desired signal and the interference signal. 


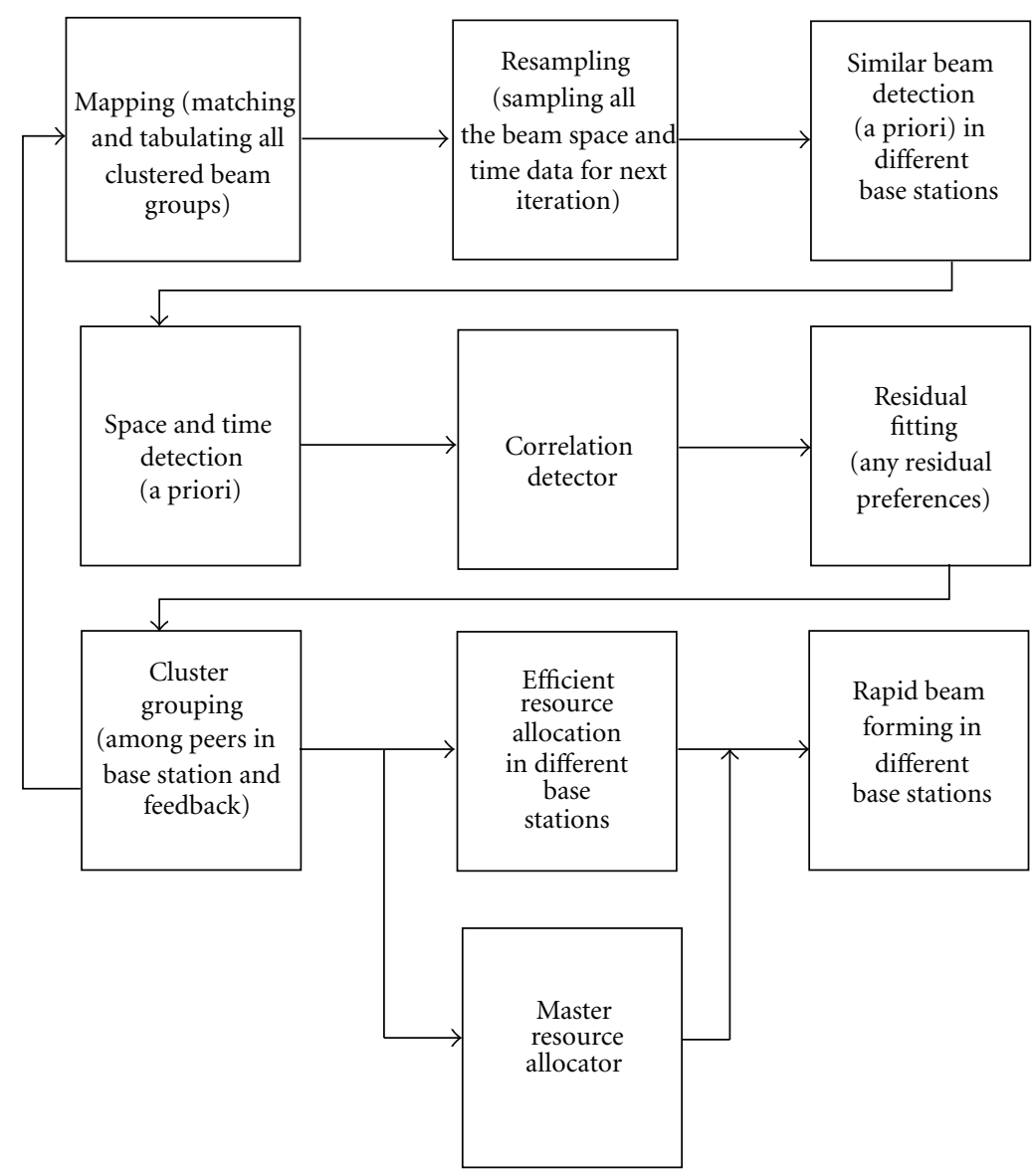

FIGURE 13: Block diagram for combinational beam-forming algorithm among peer members between different base stations.

\section{Combinational Beam-Forming Algorithm among Peer Members among Different Base Stations}

The evolution of wireless communication system requires new technologies to support better quality communications, new services, and applications. Smart antennas have become a hot topic of research employed in base stations. With a smart antenna directive beam patterns can be steered toward the desired signal and deep nulls can be formed toward the interference, thus spatial filtering is realized. This brings the benefits such as lower power transmission, higher spectrum efficiency, better link quality, and higher system capacity. Various beam-forming and direction of arrival estimation algorithms have been designed for single base station. Most of these algorithms are designed based on the digital beam forming antenna arrays. Signals received by individual antenna elements are down-converted into base band signals. These signals are digitized and fed into digital signal processing chip where the algorithms reside in. The master resource allocated decides the corresponding group as shown in Figure 13. However, radio frequency circuit branches connected to the array elements, analog-to-digital converters, and the baseband DSP chip consume a considerable amount of DC power. Furthermore, each channel connected to the array sensor has the same structure, so the cost of fabrication increases with the number of array elements.

Thanks to the recent development of GaAs monolithic microwave integrated circuit (MMIC) technologies, the beamformer could be integrated to a single chip at the RF front end instead of the baseband. The advantages are the reduced quantization errors and the increased dynamic range. However, their costs of fabrication still limit the range of limitation. All these factors make DBF and MBF antennas suitable for low power consumption and low cost systems and thus hinder the mass application of the smart antenna technologies. For example, it could be too costly to equip DBF antenna arrays at battery powered laptops or mobile computing terminals within a wireless network. Same procedure used for single base station is used except here the base stations can be more than one.

\section{Conclusion}

In this paper a rapid beam forming algorithm for smart antennas was proposed using the concepts of fractal array and novel algorithms. It was further enhanced by using successive algorithms called "Assignment of Usage Time and Location Tag Algorithm." It was found that the proposed method 
greatly reduces the time needed for calculating the array factor and the proposed new techniques still enhance the allocation of computational resources for the development of rapid beam. It also reduces the memory requirements to a greater extent. Furthermore, new combinational beamforming algorithm among peer members within a single base station and combinational beamforming algorithm among peer members in different base stations have been proposed for performance enhancement and optimization.

\section{References}

[1] B. B. Mandelbrot, The Fractal Geometry of Nature, W. H. Freeman and Company, New York, NY, USA, 1983.

[2] J. P. Gianvittorio and Y. Rahmat-Samii, "Fractal antennas: a novel antenna miniaturization technique, and applications," IEEE Antennas and Propagation Magazine, vol. 44, no. 1, pp. 20-36, 2002.

[3] S. R. Best, "The fractal loop antenna: a comparison of fractal and non-fractal geometries," in Proceedings of the IEEE Antennas and Propagation Society International Symposium, pp. 146-149, Boston, Mass, USA, July 2001.

[4] D. H. Werner and S. Ganguly, "An overview of fractal antenna engineering research," IEEE Antennas and Propagation Magazine, vol. 45, no. 1, pp. 38-57, 2003.

[5] Y. Kim and D. L. Jaggard, "The fractal random array," Proceedings of the IEEE, vol. 74, no. 9, pp. 1278-1280, 1986.

[6] A. Lakhtakia, N. S. Holter, V. K. Varadan, and V. V. Varadan, "Self-similarity in diffraction by a self-similar fractal screen," IEEE Transactions on Antennas and Propagation, vol. AP-35, no. 2, pp. 236-239, 1987.

[7] X. Liang, W. Zhenson, and W. Wenbing, "Synthesis of fractal patterns from concentric-ring arrays," IEE Electronics Letters, vol. 32, no. 21, pp. 1940-1941, 1996.

[8] C. Puente-Baliarda and R. Pous, "Fractal design of multiband and low side-lobe arrays," IEEE Transactions on Antennas and Propagation, vol. 44, no. 5, pp. 730-739, 1996.

[9] D. H. Werner, R. L. Haupt, and P. L. Werner, "Fractal antenna engineering: the theory and design of fractal antenna arrays," IEEE Antennas and Propagation Magazine, vol. 41, no. 5, pp. 37-59, 1999.

[10] M. Chryssomallis, "Smart antennas," IEEE Antennas and Propagation Magazine, vol. 42, no. 3, pp. 129-136, 2000.

[11] W. C. Lee and S. Choi, "Adaptive beamforming algorithm based on eigen-space method for smart antennas," IEEE Communications Letters, vol. 9, no. 10, pp. 888-890, 2005.

[12] M. Z. Shakir and T. S. Durrani, "Narrowband beamforming algorithm for smart antennas," in Proceedings of the 5th International Bhurban Conference on Applied Sciences and Technology (IBCAST '07), pp. 49-54, Islamabad, Pakistan, January 2007.

[13] J. Liberti and T. S. Rappaport, Smart Antennas for Wireless Communications: IS-95 and Third Generation CDMA Applications, Prentice Hall, New York, NY, USA, 1999.

[14] M. M. Badjian, K. Thirappa, T. S. Kiong, J. K. S. Paw, and P. S. Krishnan, "Coverage performance analysis of genetic algorithm controlled smart antenna system," in Proceedings of the 8th IEEE Student Conference on Research and DevelopmentEngineering: Innovation and Beyond (SCOReD '10), pp. 81-85, Putrajaya, Malaysia, December 2010.

[15] M. Levy and D. S. Kumar, "Novel algorithms for rapid beam forming in optical antennas for microwave photonics applications using smart-fractal concepts," in Proceedings of the IETE ICon Conference, October 2011.
[16] M. Levy, D. S. Kumar, A. Dinh et al., "A novelistic approach for rapid beam forming in smart antennas for wireless applications using smart-fractal concepts and new algorithm," in Proceedings of the International Conference on Advances in Mobile Network, Communication and its Applications (MNCAPPS '12), pp. 5-10, Bangalore, India, August 2012. 

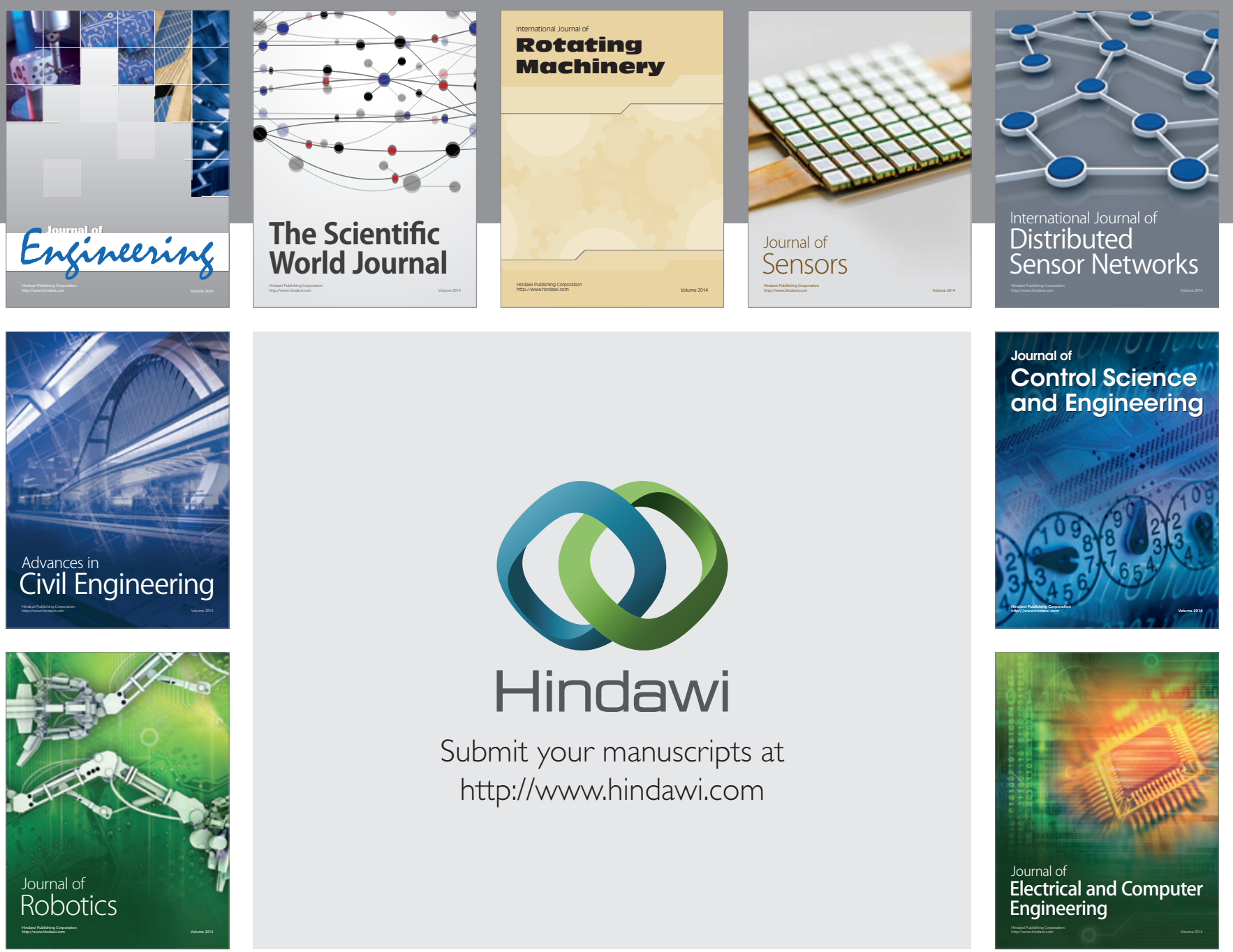

Submit your manuscripts at

http://www.hindawi.com
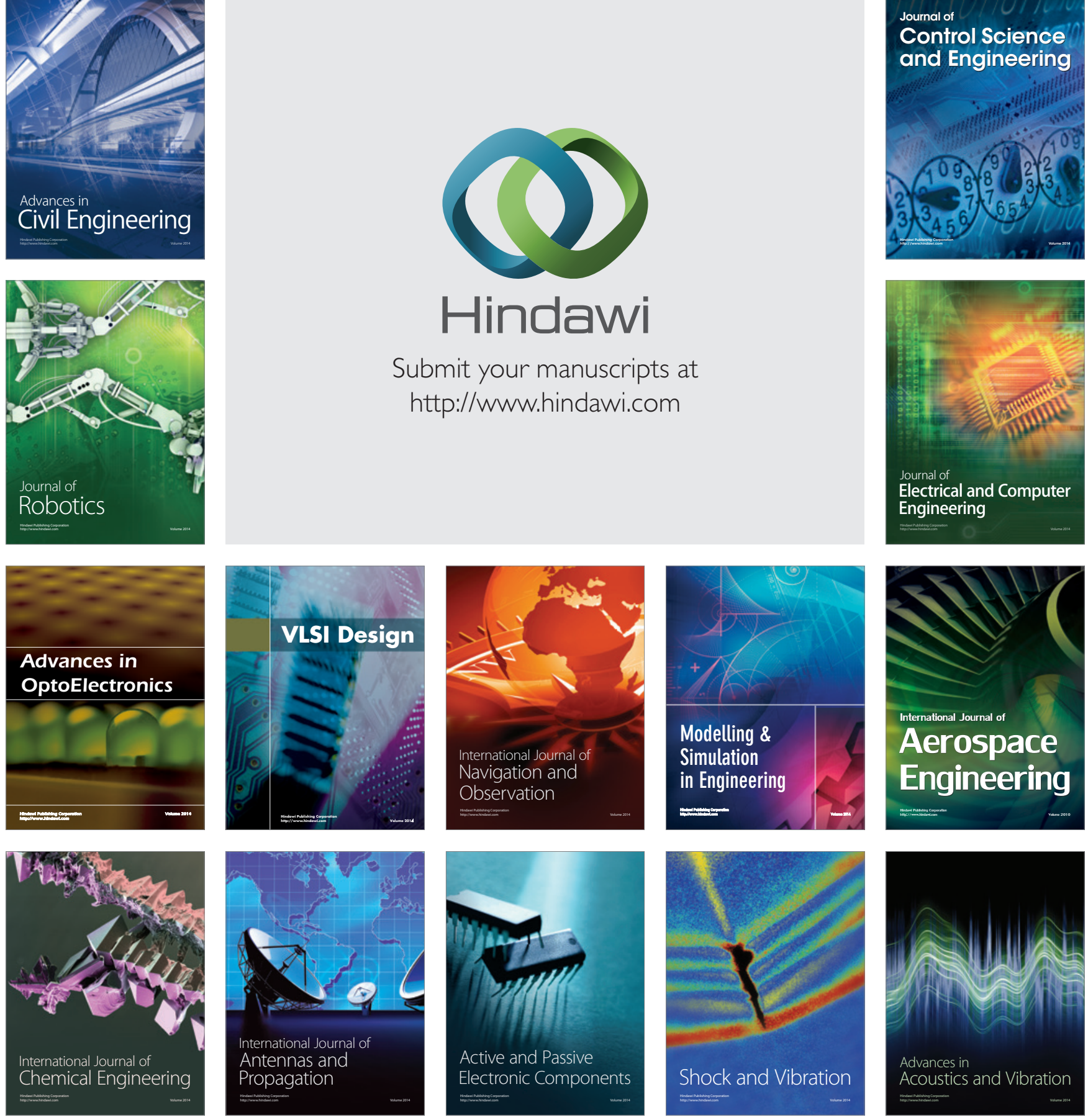